\title{
Paying the ultimate price
}

\author{
Fiona Godlee editor in chief
}

The BMJ

Around the world, health and social care workers are dying because of occupational exposure to covid-19. Many hundreds have died, including more than 100 in the UK. Are these deaths, and others yet to come, an inevitable cost of this pandemic? Not if some (or all) of them could have been avoided with better planning and provision. It is impossible not to feel let down by political and healthcare leaders who, while sloganning and clapping for the NHS, have so evidently failed to protect those working within it.

The UK government's response to this crisis has been characterised from the beginning by complacency, arrogance, and delay, worsened in subsequent weeks by broken promises about the supply of personal protective equipment, apparent ignorance of the situation on the frontline, and poorly explained and shifting guidance. Mary McCarthy and Giuseppe Rivolta write that more honesty would have helped doctors in Italy accept risks caused by the shortfall in PPE (bit.ly/2XYaTrJ). But zero harm to healthcare staff is possible, they report. This would mean dropping WHO guidance in favour of the European Centre for Disease Prevention and Control's recommendations: FFP2 respirator masks as a minimum for all staff.

John Robertson and colleagues agree (bit.ly/2VpmtdK). Until it is clear how much transmission is due to aerosol as well as droplet infection, surgical masks should not be considered effective protection, they say. They are damning about the UK government's attempts to shift the blame for staff deaths onto community infection and have no faith in its proposed investigation. "Without referring each death to the coroner, can we be confident that the circumstances of their employment have not resulted in these individuals paying the ultimate price through their daily work?"
Meanwhile, doctors are scared to speak out about inadequate protection, reports Clare Dyer (doi:10.1136/bmj.m1592). This is especially true for staff from ethnic minority backgrounds, says Chaand Nagpaul, and they are most at risk from covid-19, accounting for nine of the first 10 UK doctors who have died (bit.ly/2RTWo4m). Staff who are at high risk should be shielded by redeployment away from the front line, writes Helen Salisbury (doi:10.1136/bmj.m1572). Thankfully, this is now happening, reports Jacqui Thornton, as part of a "breathtaking" transformation of services across the NHS (doi:10.1136/bmj. m1444).

Should the public wear face masks? Trish Greenhalgh and colleagues conclude that, while good evidence is lacking, policy makers should apply the precautionary principle (doi:10.1136/ bmj.m1435). Concern that masks should be kept for use by health workers is "an argument for manufacturing more masks, not for denying them to the populations who could potentially benefit."

The precautionary principle should apply even more to anyone whose work puts them in harm's way during this pandemic. First, the guidance should be set to achieve zero harm. Second, our leaders must be honest and accountable when lack of adequate supply makes this impossible, with any emergency measures being openly debated and explained. Third, we must shield staff who are most at risk through age, sex, ethnic origin, and comorbidities. Finally, so that we can learn for the future, honour the sacrifice, and seek compensation for families, all deaths of health and social care workers should be referred to the coroner for independent review. 